\title{
A Novel Design Approach for Beam Bridge Structure Pressure Sensor Base on PZT- 5A Piezoelectric
}

\author{
Maibam Sanju Meetei ${ }^{1, *}$, Aheibam Dinamani Sihgh ${ }^{2}$ and Swanirbhar Majumder ${ }^{3}$ \\ ${ }^{1}$ Rajiv Gandhi University \\ ${ }^{2}$ Dept. of Electronics and Communication, National Institute of Technology, Imphal \\ ${ }^{3}$ Dept. of Information and Technology, Tripura University, Agartala \\ Received 19 August 2020; Accepted 20 January 2021
}

\begin{abstract}
With the spread of the Internet of things, the smart sensors with low power consumption, high sensitivity and can operate in harsh condition are required for various applications. Piezoelectric based sensors are the better as the sensors operate with high linearity with a wide range of mechanical input, and immune to electromagnetic fields and radiations. It is difficult to expedite for developing such sensors as the literature is widely dispersed over many disciplines. In this study, a new approached of mathematical modelling for PZT-5A piezoelectric base bridge structure pressure sensor has been studied. For validation of the mathematical model, a 3D model of a sensor is designed and simulated with the COMSOL multiphysics simulator. Various factors affecting the output voltages and sensitivity of the sensor described in mathematical modelling. Two parts of mathematical modelling consider mechanical modelling and electrostatic modelling. Mechanical modelling enables to analyse the mechanical stress developed on the surface of the sensor. Electrostatic modelling computes various operating modes of the piezoelectric and output voltage of the sensor. Transverse mode of operation for piezoelectric is applicable for bridge structure designed. The electrical equivalent circuit of the sensor is discussed. Comparative studies are done between the mathematical modelling values and simulated output values. From the comparative studies, the output behaviour and various factors affecting the output of a sensor are observed. The sensor mechanical stresses are linear in magnitude with a positive slope to the applied pressures. The sensor output voltages are linear in magnitude with a negative slope to the applied pressures. Tensile stress and negative voltage are developed on the top of the surface. The sensitivities of the sensor are high and the values are $-01.957 \mathrm{mV} / \mathrm{kPa}$ and $-1.7 \mathrm{mV} / \mathrm{kPa}$ for calculating and simulated respectively.
\end{abstract}

Keywords: displacement function, neutral plain, operational mode, sensitivities, stress.

\section{Introduction}

Various types of Micro-Electro Mechanical Systems (MEMS) pressure sensors are developed and many of them are available in the market and mass-produced. For a researcher or designer, it is difficult to expedient in developing the MEMS pressure sensor. Based on the sensing mechanism, there are various types of pressure sensors. They are

(i) Resistive: this sensor is generally piezo-resistive pressure sensor which changes the resistance to deformation [1][2].This sensor has a limitation which sensitivity is reduced because the strain is reduced as the scaling in size of the sensor,

(ii) Capacitive: this sensor changes its electrical capacitance by the deflection of a diaphragm [3][4]. This sensor exhibit a nonlinear as capacitance is inversely proportional between the gaps,

(iii) Inductive: this sensor work on the principle of magnetic field induction [5][6]. The Main limitation is scaling and magnetic interference due to metal,

(iv)Thermal: this sensor work on the principle of change in thermal conductivity due to change in density by

*E-mail address: maibamhamam@gmail.com

ISSN: 1791-2377 @ 2021 School of Science, IHU. All rights reserved.

doi:10.25103/jestr.141.23 measure pressure [7]. The main drawback is system complexity,

(v) Optical: this sensor utilized the concept of Fibre Bragg Gratings [8]. This sensor used when there is a need for high immune to electromagnetic interferences, and

(vi) Piezoelectric: this sensor is working on the principle of piezoelectricity and used for measuring dynamic pressure [9][10][16].

All the sensors are active sensor which requires external power sources, except the piezoelectric sensor, which doesn't require any external power sources. Such piezoelectric sensors remove the struggle of engineers with wires and batteries for monitoring the pressure and it has high sensitivity. These piezoelectric sensors are used for sensing vibration or pressure with low or high frequency. These sensors appear hysteresis as an offset which depends on a field level, cycle time and material used. This hysteresis is considerable for high frequency greater than $1 \mathrm{kHz}$ as heat accumulation aftermeach cycle of operation [11].

Piezoelectricity is the property of certain dielectric material to produce electric charges when mechanical deformation or physically deformed in the presences of electric fields [11]. This phenomenon is due to spontaneous polarization caused by the displacement of electrons relative to the atomic centre, so piezoelectric materials are used for sensing continuous varying physical quantity. Because of this 
spontaneous nature, piezoelectric materials are used for sensing the vibrations. Piezoelectric sensors have excellent linearity with a wide range of mechanical input, wide ranges of operating frequency, and immune to electromagnetics, radiation and operate in harsh condition [12]. Piezoelectric sensors have low power consumption, high sensitivity and are widely used in various applications as the Internet of Things is widely spread.

For piezoelectric there are three basic modes of designing of sensor depending on the direction of induced strain and induced voltage. They are longitudinal mode, transverse mode and shear mode. Depending on the modes, the voltagecoefficient $(d x y)$ values are also different for the individual piezoelectric material as the directions of deformation are different.

Longitudinal $\left(\mathrm{d}_{33}\right)$ mode: In this mode, the direction of the applied forces is along the direction of the strain and induced charges as shown in Fig 1.

Transverse $\left(d_{31}\right)$ mode: In this mode, the direction of the applied force is perpendicular to the induced strain and along with the induced charges as shown in Fig. 2.

Shear $\left(d_{15}\right)$ mode: In this mode, the direction of the forces and the induced strain are inclined, but the applied force and induced charges are perpendicular as shown in Fig.3.

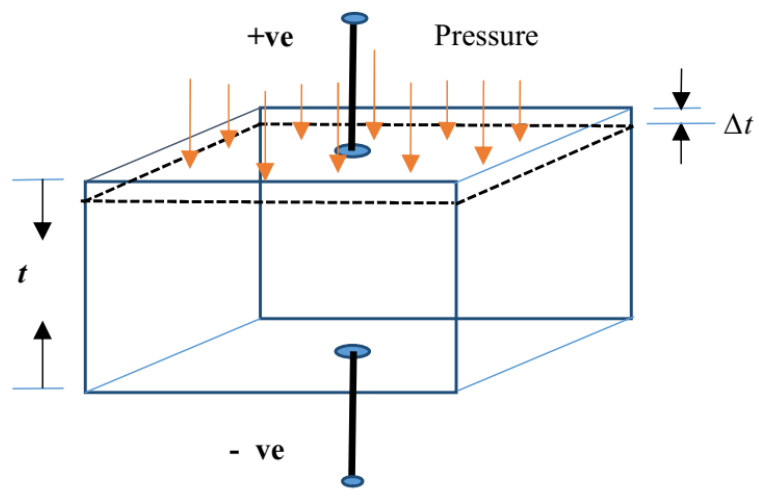

Fig. 1. Longitudinal $\left(d_{33}\right)$ mode

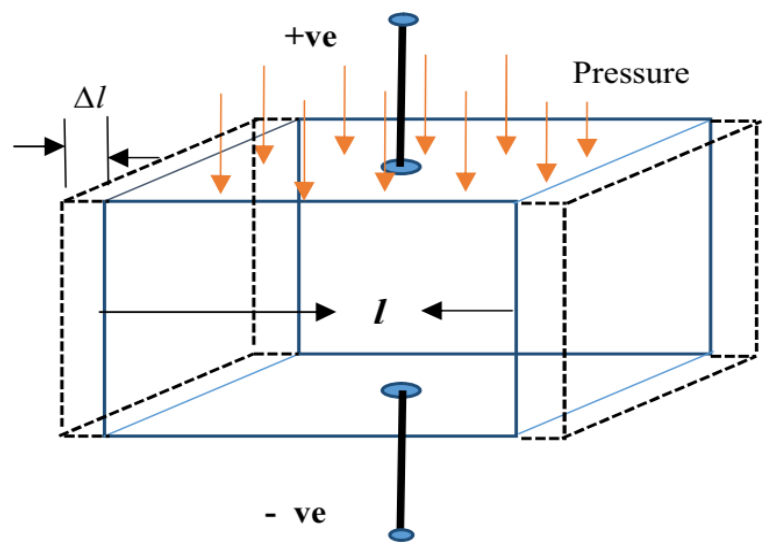

Fig. 2. Transverse $\left(d_{31}\right)$ mode.

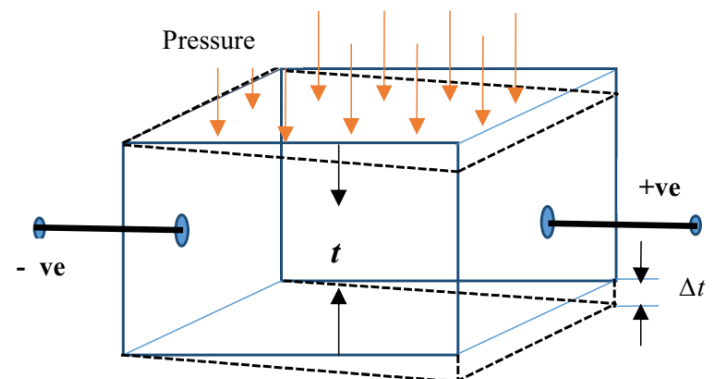

Fig. 3. Shear $\left(d_{15}\right)$ mode.
In prior studies, piezoelectric sensors are developed and marketed. But for a designer, it is difficult to develop the literature as the design principles are widely depressed over many disciplines. In this study, we develop the fundamental mathematical model of the piezoelectric pressure sensor with the bridge structure for low-pressure sensing. This paper has a major four-part. First, Mathematical modelling of the sensor, explains the derivation of the mechanical stress and the output voltage induces due to applied pressure in details. The various three modes of operations of piezoelectric material have been explained in detail. Second, 3D model analysis using COMSOL, this partially explains the 3D structure of the sensor and simulation of the sensor and analyses the output. Third, Comparative analysis between simulation and mathematical model, this part perform a comparison between the mathematical and the simulated output of mechanical stress and output voltage of the sensor. A comparison of mathematically calculated values and simulated values for mechanical sensitivity and electrostatic sensitivity is carried out. After various comparisons, various observations are made for the conclusion. Finally, Conclusion, the sensitivity of the sensor and from various observations, many the factors affecting the sensitivity are concluded.

\section{Mathematical modelling of the sensor}

\subsection{Mechanical modelling}

For a beam bridge pressure sensor, it can be considered that uniform pressure is applied at the top surface as the size is very small. This pressure will create a deflection which induces stress and strain on beam. The induced stress is directly proportional to the output voltage in the magnitude of the sensor. A negative voltage is developed on the surfaces where tensile stress is experienced and a positive voltage is induced on the surface where compressive stress is experienced. To find out the stress, let us consider the displacement function in Eq. 1 of the beam bridge structure for Fig. 4. [13].

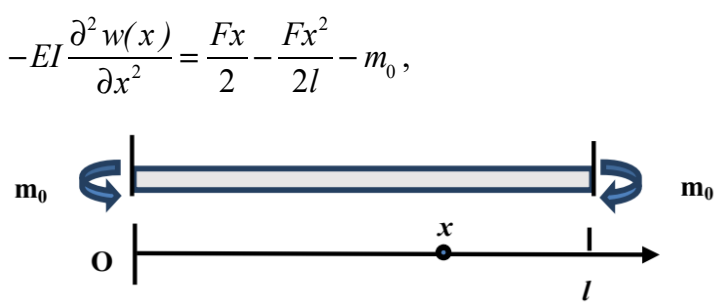

Fig. 4. Bridge Structure.

where,

$E$ : Young's Modulus, $I$ : moment of inertia, $x$ : position on the beam, $\frac{\partial^{2} w(x)}{\partial x^{2}}$ : double derivative of the deflection of a beam with respect to $x, \mathrm{~F}$ : the total force applied on the surface, $l$ : length of the beam and $m_{0}$ : moment at the clamped.

The applied force surface area is very small, so it can be considered as a uniformly distributed load with a pressure. The uniform force or load is given

$$
\begin{aligned}
& F=P A . \\
& A=l b .
\end{aligned}
$$

where, 
P: uniformly applied pressure, A: surface area, $b$ : breath of the beam.

Integrating Eq. 1 with respect to $x$ can be written as below

$-E I \frac{\partial w(x)}{\partial x}=\frac{F x^{2}}{4}-\frac{F x^{3}}{6 l}-x m_{0}$

Considering the boundary condition at $x=l$ in Eq. 4, the value of

$m_{0}=\frac{F l}{12}$.

Integrating the Eq. 4 and putting the value of $\mathrm{m}_{0}$. The equation can be written as follows

$$
\begin{aligned}
& -E I w(x)=\frac{F x^{3}}{12}-\frac{F x^{4}}{24 l}-\frac{F l x^{2}}{24} \\
& w(x)=\frac{(l-x)^{2} F x^{2}}{24 I E l} .
\end{aligned}
$$

The deflection $w(x)$ of the beam in $x$ position is given by Eq. 7. The maximum deflection is occurring in the centre of the beam, i.e. $x=(l / 2)$ so, the maximum deflection equation is given as follows

$$
w(x)_{\max }=\frac{F l^{3}}{384 E I} .
$$

The moment of inertia of a rectangular beam is given by the following equation

$$
I=\frac{b h^{3}}{12}
$$

where, $h$ : the thickness of the beam.

Eq. 8 can be written after putting the value of $I$ as follows

$$
w(x)_{\max }=\frac{F l^{3}}{32 E b h^{3}} .
$$

In Minhang Bao, 2005, the stress equation of a beam is given by,

$$
T(x)=-E z \frac{\partial^{2} w(x)}{\partial x^{2}},
$$

where,

$z$ : distance of the point $x$ from the natural plane of the beam. When applying pressure on the beam stress is induced inside the whole body of the beam, but a plane where minimum stress is there inside the beam is called a natural plane [13]. The position of the natural plane varies with the thickness of the multi-layers, Young's Modulus of the materials and the structure. For a single layer, a natural plane of the beam or cantilever is the mid-plane of the structure. Equation 1 can be written as

$$
\frac{\partial^{2} w(x)}{\partial x^{2}}=-\left(\frac{F x}{2}-\frac{F x^{2}}{2 l}-m_{0}\right) \frac{1}{E I}
$$

Putting the value of $m_{0}$ and $I$ on in Eq.12

$$
\frac{\partial^{2} w(x)}{\partial x^{2}}=-\left(\frac{F x}{2}-\frac{F x^{2}}{2 l}-m_{0}\right) \frac{12}{E b h^{3}}
$$

$$
\frac{\partial^{2} w(x)}{\partial x^{2}}=-F\left(6 x l-6 x^{2}-l^{2}\right) \frac{1}{E l b h^{3}}
$$

Eq. 11 can be written after putting the value $\frac{\partial^{2} w(x)}{\partial x^{2}}$,

$$
T(x)=\frac{z F\left(6 x^{2}-6 x l+l^{2}\right)}{l b h^{3}}
$$

For a single layer, the maximum stress occurs at $x=l$ and $x=0$ on the top of the surface. For single-layer $z=h / 2$ but for multi-layer, first, it needs to find the neutral plain i.e. $z_{0}$. The distance between the $z_{0}$ and the position of $\mathrm{x}$ where the value of $z$. Eq. 14 can be rewritten to find the stress is as follows

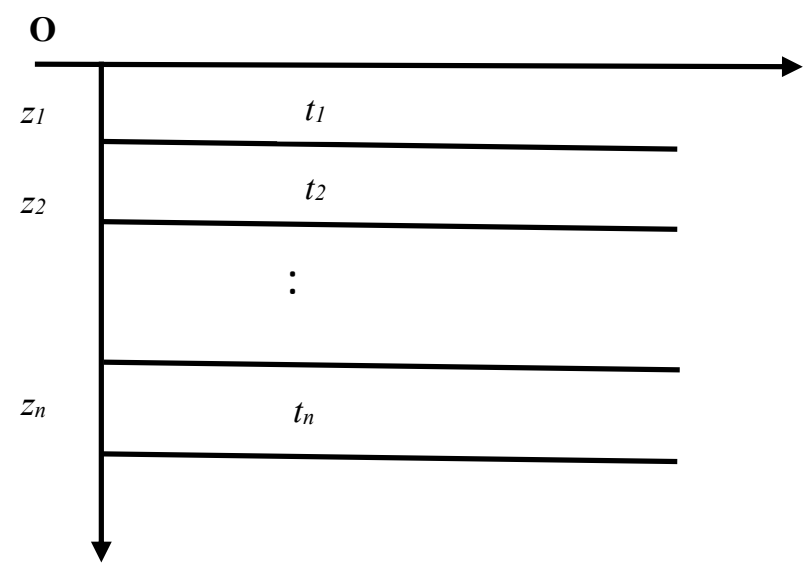

Fig. 5. Showing a multilayer system

In [13], the position of neutral plain $z_{0}$ having $n$ layers can be determined by

$$
z_{0}=\frac{E_{11} t\left(0+z_{1}\right)+E_{22} t\left(z_{1}+z_{2}\right)+\ldots+E_{n n} t\left(z_{n-1}+z_{n}\right)}{2\left(E_{11} t+E_{22} t+\ldots+E_{n} t\right)},
$$

where, $E_{1}, E_{2} \ldots E_{n}$ are the Young's modulus of the layers with $t_{1}, t_{2} \ldots t_{n}$ are the thickness of the layers respectively. And the value of $z_{n}$ commutative thickness of the layers given by

$$
z_{i}=\sum_{j=1}^{i} t_{j} \quad(i=1,2,3 \ldots \ldots n) .
$$

\subsection{Electrostatic Modelling}

In the piezoelectric sensor, the charges are developed on opposite sides of the piezoelectric surface which depends on the operation mode of the sensor. These charges developed a potential difference between the surfaces, and these two surfaces behave like a capacitor. The equivalent circuit diagram can be shown in Fig.6. 


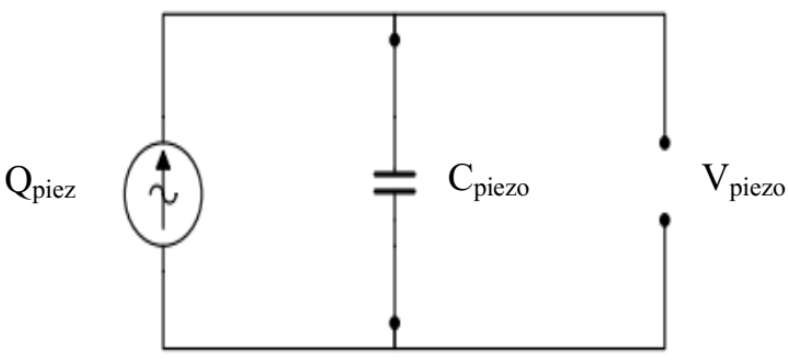

Fig. 6. Equivalent Circuit Diagram of a piezoelectric pressure sensor. 19.

The voltage of the circuit can be calculated as given in Eq.

$V=Q / C$,

where, $V$ is the output voltage, $\mathrm{Q}$ is the charges developed and $\mathrm{C}$ is the capacitance between the two surfaces.

For the bridge's structure, the operation mode is the transverse mode. So electrostatic charge developed due to the induced stress for transverse mode is given by Eq. 15 [14].

$q(x)=T(x, y) d_{31}$,

where,

$d_{3 l}$ is the strain constant of piezoelectric material, and $q(x)$ is the charge developed on the surface at position $x$ or charge density. For bridge structure, stress is a function of $x$ only so $T(x, y)$ can be replaced by $T(x)$.

$q(x)=T(x) d_{31}$,

The total charge developed on the surface is given by

$$
\begin{aligned}
& Q(x)=b l_{\text {pizo }} q(x), \\
& Q(x)=b l_{\text {pizo }} T(x) d_{31},
\end{aligned}
$$

Where

$Q(x)$ is the total charge developed on the surface of the piezoelectric layer, $l_{p i z o}$ is the length of the piezoelectric layer.

So the capacitance developed between the two surfaces is given by Eq. 22 .

$$
\begin{aligned}
& C=\frac{b l_{\text {pizo }} T(x) d_{31}}{V(x)}, \\
& \frac{\varepsilon_{33} A_{\text {pizo }}}{t}=\frac{b l_{\text {pizo }} T(x) d_{31}}{V(x)},
\end{aligned}
$$

where,

$\varepsilon_{33}$ is the permittivity of the piezoelectric material. Since the $A_{\text {pizo }}$ is an area of the piezoelectric layer, $t$ is the thickness of the piezoelectric and $V(x)$ is the output voltage of the piezoelectric.

$$
\varepsilon_{33}=\frac{d_{31}}{g_{31}} .
$$

where,

$g_{3 l}$ is the voltage constant of piezoelectric material.

Putting the value of $d_{31}$ in Eq. 26, the output voltage of the piezoelectric in transverse mode is given by

$$
V(x)=T(x) \operatorname{tg}_{31} \text {, }
$$

In MEMS devices, there are two sensitivity, i.e. mechanical sensitivity (SM) and electrostatic sensitivity (SE). In this study, the mechanical sensitivity is the ratio of change in stress to change in applied pressure given as

$S_{M}=\frac{\Delta T(x)}{\Delta P}$

where,

$\Delta T x()$ is the change in stress, and $\Delta P$ is the change in applied pressure. The electrostatic sensitivity is the ratio of change in output voltage to change in applied pressure.

$S_{M}=\frac{\Delta V(x)}{\Delta P}$

where,

$\Delta V(x)$ is the change in output voltage for position $x$.

\section{3D design and simulation}

\subsection{Structure and material of the sensor}

In Simulation, COMSOL Multiphysics simulator base on finite element method (FEM) is used for various analyses of the 3D model of the sensor.

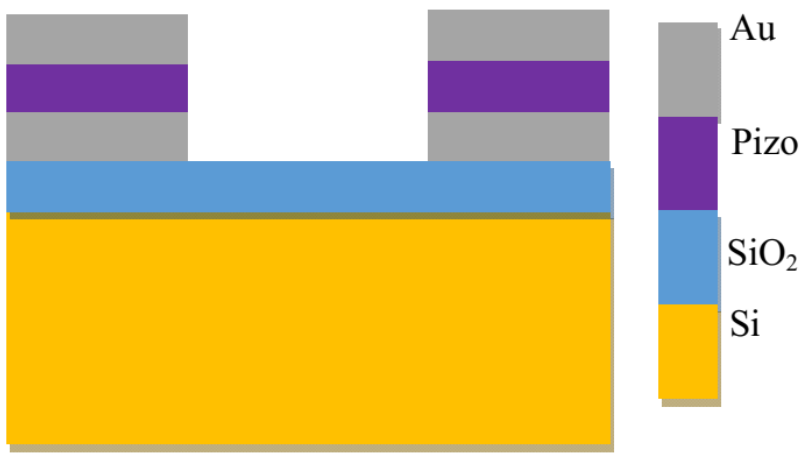

Fig. 7 D Model of the piezoelectric bridge structure pressure sensor.

This 3D model of sensor is a bridge structure with Silicon (Si) as the mechanical structure, Silicon-dioxide ( $\mathrm{SiO} 2)$ as an insulator between $\mathrm{Si}$ and Gold $(\mathrm{Au})$. A piezoelectric layer is taken as a sensing layer between the two Gold electrodes. In COMSOL, 3D model of design for simulation and analysis as shown in Fig. 7. In Tab. 1, the dimensions of the designed structure are tabulated.

For this study, piezoelectric PZT-5A is taken for analysis. The strain constant, $d 31$, and voltage constant, $g 31$, are -190 $\mathrm{x} 10^{-12} \mathrm{~m} / \mathrm{V}$ and $-13.7 \times 10^{-3} \mathrm{Vm} / \mathrm{N}$ respectively [15].

Table 1. Dimensions of the sensor

\begin{tabular}{c|c|c|c}
\hline \multirow{2}{*}{ Material } & \multicolumn{3}{|c}{ Dimension } \\
\cline { 2 - 4 } & Length & Breath & $\begin{array}{c}\text { Thickness/ } \\
\text { Height }\end{array}$ \\
\hline Silicon (Si) & $300 \mu \mathrm{m}$ & $40 \mu \mathrm{m}$ & $20 \mu \mathrm{m}$
\end{tabular}


Maibam Sanju Meetei, Aheibam Dinamani Sihgh and Swanirbhar Majumder/

Journal of Engineering Science and Technology Review 14 (1) (2021) 193 - 199

\begin{tabular}{c|c|c|c}
$\begin{array}{c}\text { Silicondioxide } \\
\left(\mathrm{SiO}_{2}\right)\end{array}$ & $300 \mu \mathrm{m}$ & $40 \mu \mathrm{m}$ & $3 \mu \mathrm{m}$ \\
Gold $(\mathrm{Au})$ & $50 \mu \mathrm{m}$ & $40 \mu \mathrm{m}$ & $3 \mu \mathrm{m}$ \\
$\mathrm{PZT}-5 \mathrm{~A}$ & $50 \mu \mathrm{m}$ & $40 \mu \mathrm{m}$ & $5 \mu \mathrm{m}$ \\
\hline
\end{tabular}

For the analysis, a pressure range of $1 \mathrm{kPa}$ to $20 \mathrm{kPa}$ with a step size of $1 \mathrm{kPa}$ has been taken as input pressures.

\subsection{Simulation Output}

Mechanical Stress and Output Voltage are two very important parameters for the analysis. The mechanical stress distribution for the applied pressure $20 \mathrm{kPa}$ on the beam structures is illustrated as in Fig. 8. The maximum stress is developed in near the edge of clamping and gradually decrease toward the centre. The electrostatic voltage distribution for the applied pressure $20 \mathrm{kPa}$ on the beam structures is illustrated as in Fig. 9. The positive voltages are developed at compressive strain are developed and negative voltages are developed at tensile stress are developed.

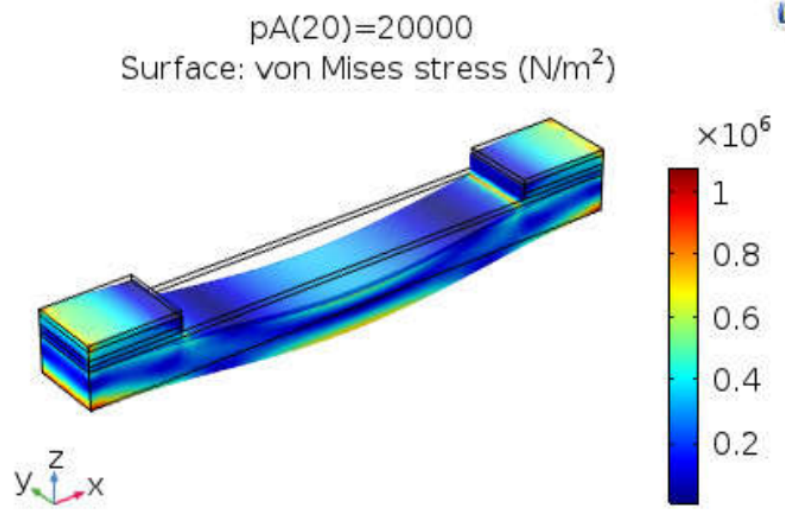

Fig. 8. Stress distribution on the sensor. $\operatorname{pA}(20)=20000$ surface: Electric potential $(V)$

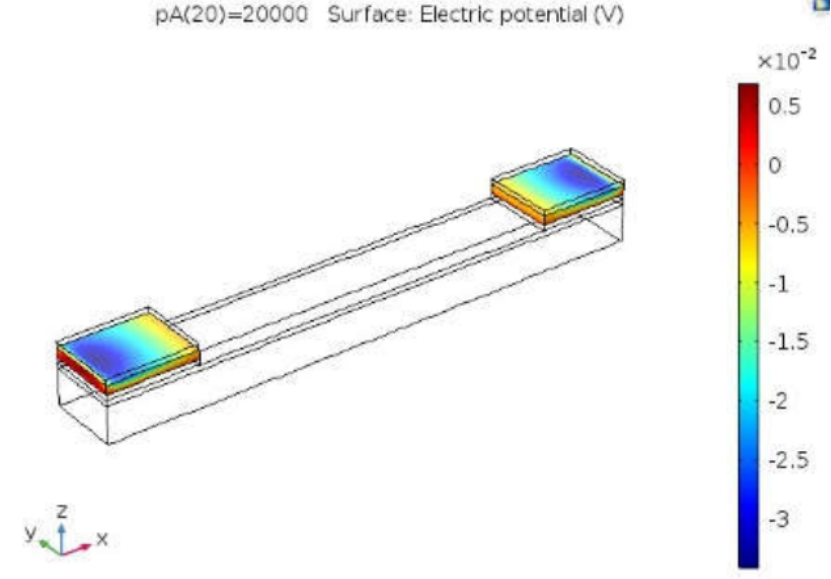

Fig. 9. Voltage distribution on the sensor.

\section{Comparative studies calculated and simulated}

A comparison table for the analytical mechanical stress and simulated mechanical stress are tabulated in Tab. 2. For mathematical calculation, the approximated value of $z=-15.5$ $\mu \mathrm{m}$.

Table 2. Applied Pressures vs. Mechanical Stresses of the sensor

\begin{tabular}{c|c|c}
\hline \multirow{2}{*}{$\begin{array}{c}\text { Applied } \\
\text { Pressure } \\
{[\mathbf{k P a}]}\end{array}$} & \multicolumn{2}{|c}{ Mechanical Stress } \\
\cline { 2 - 3 } & Calculated [kPa] & Simulated [kPa] \\
\hline 1 & 35.49 & 35.39 \\
2 & 70.98 & 72.70
\end{tabular}

\begin{tabular}{c|l|l}
3 & 106.47 & 107.07 \\
4 & 141.97 & 143.60 \\
5 & 177.46 & 179.05 \\
6 & 212.95 & 215.09 \\
7 & 248.44 & 250.25 \\
8 & 283.94 & 284.37 \\
9 & 319.43 & 321.08 \\
10 & 354.92 & 355.18 \\
11 & 390.41 & 392.07 \\
12 & 425.91 & 428.41 \\
13 & 461.40 & 465.37 \\
14 & 496.89 & 498.08 \\
15 & 532.38 & 535.23 \\
16 & 567.88 & 567.05 \\
17 & 603.37 & 604.37 \\
18 & 638.86 & 636.32 \\
\cline { 1 - 1 } 19 & 674.35 & 675.78 \\
20 & 709.85 & 710.76 \\
\hline
\end{tabular}

From Tab. 2, the mechanical sensitivity of the designed sensor is

$$
\begin{aligned}
& S_{M}=\frac{\Delta T(x)}{\Delta P} \\
& \left(S_{M}\right)_{\text {analytical }}=\frac{709.85-35.49 \mathrm{kPa}}{20-1 \mathrm{kPa}}=35.49,
\end{aligned}
$$

Similarly,

$$
\left(S_{M}\right)_{\text {simulated }}=\frac{710.76-35.39 \mathrm{kPa}}{20-1 \mathrm{kPa}}=35.54,
$$

The analytical and simulated mechanical sensitivity of the sensor is 35.49 and 35.54 respectively, which are very close to each other.

The stress developed on the surface is tensile stress as the value of the stress is positive. In the graphical representation of Tab. 2 as shown in Fig. 10, the analytical stress value and simulated stress value are overlapped with each other. The mechanical stress induces on the bridge are linearly proportional to the input applied pressure in magnitude. The slope of the graph is positive. It shows the derived Eq. of stress, i.e. Eq. (16) can be implemented for finding out the stresses induced in the bridge structure.

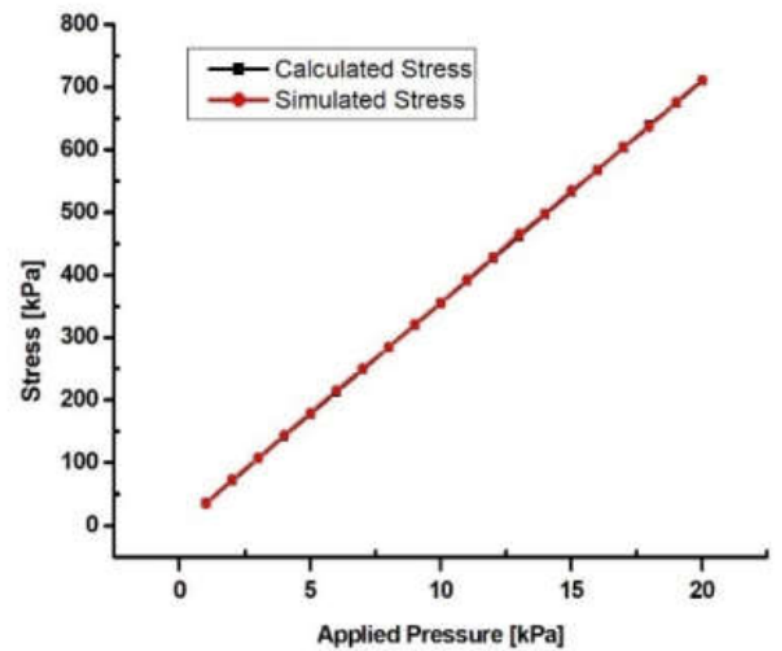

Fig. 10. Applied Pressure vs. Mechanical Stress of the sensor. 
Similarly, a comparative study for analytical voltages and simulated voltages is done. The comparative analyses tabulated in Tab. 3.

Table 3. Applied pressure vs Output voltage.

\begin{tabular}{c|c|c}
\hline $\begin{array}{c}\text { Applied } \\
\text { Pressure } \\
{[\text { kPa] }}\end{array}$ & \multicolumn{2}{|c}{ Voltages } \\
\cline { 2 - 3 } Calculated (V) & Simulated (V) \\
\hline 1 & -0.0020 & -0.0017 \\
3 & -0.0039 & -0.0034 \\
4 & -0.0059 & -0.0050 \\
5 & -0.0078 & -0.0067 \\
6 & -0.0098 & -0.0084 \\
7 & -0.0118 & -0.0101 \\
8 & -0.0137 & -0.0117 \\
9 & -0.0157 & -0.0135 \\
10 & -0.0176 & -0.0153 \\
11 & -0.0196 & -0.0169 \\
12 & -0.0216 & -0.0187 \\
13 & -0.0235 & -0.0203 \\
14 & -0.0255 & -0.0220 \\
15 & -0.0274 & -0.0237 \\
16 & -0.0294 & -0.0254 \\
17 & -0.0314 & -0.0271 \\
18 & -0.0333 & -0.0289 \\
19 & -0.0353 & -0.0305 \\
20 & -0.0373 & -0.0323 \\
& -0.0392 & -0.0340 \\
\hline
\end{tabular}

The graphical representation of Tab. 3 is shown in Fig. 11.

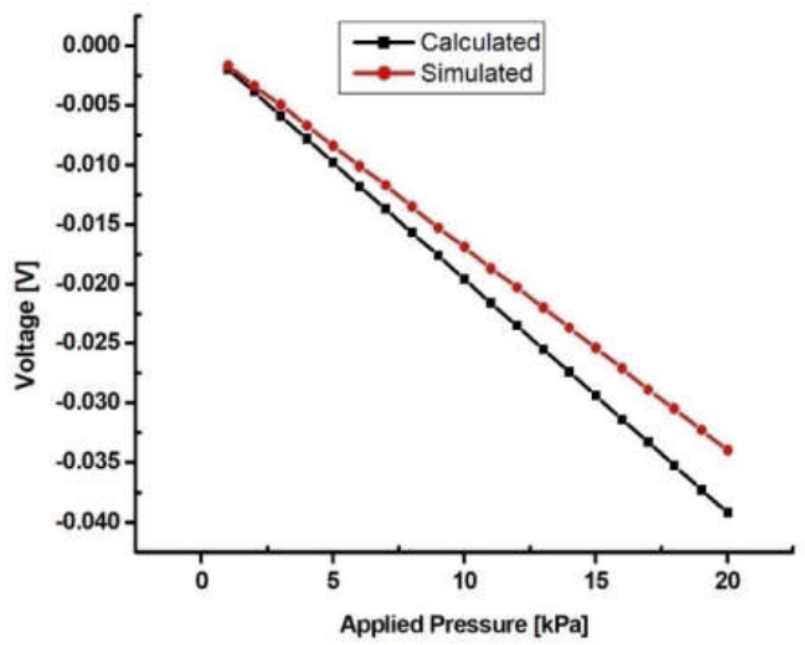

Fig. 11. Applied pressures vs. Output Voltages of the sensor.

From Tab. 3, the electrostatic sensitivity of the designed sensor is

$$
\begin{aligned}
& S_{E}=\frac{\Delta V(x)}{\Delta P}, \\
& \left(S_{E}\right)_{\text {analytical }}=\frac{(-0.0392)-(-0.0020)}{20-1} / \mathrm{kPa} \\
& =-1.957 \mathrm{mV} / \mathrm{kPa},
\end{aligned}
$$

Similarly,

$$
\begin{aligned}
& \left(S_{E}\right) \text { simulated }=\frac{(-0.0340)-(-0.0017)}{20-1} / \mathrm{kPa} \\
& =-1.7 \mathrm{mV} / \mathrm{kPa},
\end{aligned}
$$

The analytical and simulated electrostatic sensitivity of the sensor is $-1.957 \mathrm{mV} / \mathrm{kPa}$ and $-1.7 \mathrm{mV} / \mathrm{kPa}$ respectively. The deviation between the two sensitivities is very small. The voltages develop on the surface is negative as the stress is tensile. Positive voltages will be developed for compressive stress. The electrostatic output voltages of the sensor are linearly proportional to the input applying pressure in magnitude. The slope of the graph is negative. It shows the derived Eq. of electrostatic output voltage, i.e. Eq. 27 can be implemented for finding out electrostatic voltage develop on the sensor. For this design, the mechanical sensitivities are 35.49 and 35.54 for calculating and simulated respectively. The electrostatic sensitivities are $-1.957 \mathrm{mV} / \mathrm{kPa}$ and -1.7 $\mathrm{mV} / \mathrm{kPa}$ for calculating and simulated respectively.

\section{Conclusion}

In this present work, mathematical modelling and simulation are done for designing a pressure sensor base on the piezoelectric beam bridge structure. In mathematical modelling, two steps,

Mechanical modelling and electrostatic modelling have described in details. Mechanical modelling, the equation for stress is derived as the stress is directly proportional to the output voltage of the sensor. Electrostatic modelling, mode of operation of piezoelectric are discussed and derived the Eq. of the output voltage base on the equivalent circuit model of the sensor. A 3D model of a sensor is designed in COMSOL for simulation and validation of the mathematical model Eq.s. The various factors affecting the sensitivity of the stress are Young's Modulus, length, breadth, and height of the sensor structure. The various factors affecting the output voltage are thickness, placement position, voltage coefficient and operating mode of the piezoelectric layer, and stress. A comparison between the simulated and mathematically calculated is done and various observations are made. In various observations, that the mathematical stress and simulated stress are almost same and linearly varies in magnitude with a positive slope to the input pressure. But the output voltages linearly vary in magnitude with a negative slope to the input voltage. It is also observed that the positive voltages are produced where the tensile stress is induced and a positive voltage is produced where the compressive stress is induced. The sensitivities of the designed sensor are -2 $\mathrm{mV} / \mathrm{kPa}$ and $-1.7 \mathrm{mV} / \mathrm{kPa}$ for calculating and simulated respectively.

This is an Open Access article distributed under the terms of the Creative Commons Attribution License.

\section{References}

1. D. Alveringh, T.V.P. Schut, R.J. Wiegerink, W. Sparreboom, J.C. Lötters, "Resistive Pressure Sensors Integrated With A Coriolis
Mass Flow Sensor", 19th International Conference on Solid-State Sensors, Actuators and Microsystems (Transducers), (2017). 
Maibam Sanju Meetei, Aheibam Dinamani Sihgh and Swanirbhar Majumder/

Journal of Engineering Science and Technology Review 14 (1) (2021) 193 - 199

2. D. Chen, Y. Cai and M. Huang, "Customizable Pressure Sensor Array: Design and Evaluation”, IEEE Sensors Journal. pp. 63376344, (2018).

3. Darryl P. J. Cotton, Ingrid M. Graz, and Stéphanie P. Lacour, "A Multifunctional Capacitive Sensor for Stretchable Electronic Skins" IEEE SENSORS JOURNAL. pp. 2008-2009, (2009).

4. Li Chen and MehranMehregany, "A Silicon Carbide Capacitive Pressure Sensor For High Temperature And Harsh Environment Applications", Transducers 2007 - 2007 International Solid-State Sensors, Actuators and Microsystems Conference, (2007).

5. Ezzat G. Bakhoum and Marvin H. M. Cheng, "High-Sensitivity Inductive Pressure Sensor", IEEE Transactions on Instrumentation and Measurement. pp. 2960-2966, (2011)

6. W. Yang, Q. Yang, R. Yan, W. Zhang, X. Yan, F. Gao, and W. Yan, "Dynamic Response of Pressure Sensor With Magnetic Liquids", IEEE Transactions On Applied Superconductivity. pp. 18601863, (2010)

7. C. Ghouila-Houri, R. Viard, Q. Gallas, E. Garnier, A. Merlen, A.Talbi, P.Pernod, "Robust thermal microstructure for designing flow sensors and pressure sensors", IEEE SENSORS, (2017).

8. John William Arkwright, Ian David Underhill, Simon A. Maunder, Alireza Jafari, Nick Cartwright, Charles Lemckert, "Fiber Optic Pressure Sensing Arrays for Monitoring Horizontal and Vertical Pressures Generated by Traveling Water Waves", IEEE Sensors Journal. pp. 2739-2742 (2014).
9. L. Li, L. -. Wang, L. Qin and Y. Lv, "The theoretical model of 1-32 piezocomposites", IEEE Transactions On Ultrasonics, Ferroelectrics, And Frequency Control, pp. 1476-1482, (2009).

10. A. Mezheritsky, "Q-Factor Spectrum of a Piezoceramic Resonator and Method for Piezoelectric Loss Factor Determination", IEEE Transactions on Ultrasonics, Ferroelectrics, And Frequency Control, pp. 1849-1856, (2017).

11. Rob Carter and Richard Kensley, https://piezo.com/pages/introtopiezoelectricity, Acessed 12 June 2020.

12. Yongke Yan, Jie E. Zhou, Deepam Maurya, Yu U. Wang and ShashankPriya, "Giant piezoelectric voltage coefficient in grainoriented modified PbTiO3 material", Nature Communications. pp. 1-9 (2016).

13. MinhangBa, "Analysis and Design Principles of MEMS Devices", Shanghai, China: Elsevier Science (2005).

14. Ingo Kuehne, DjordjeMarinkovic, Gerald Eckstein, Helmut Seidel, "A new approach for MEMS power generation based on a piezoelectric diaphragm", Sensors and Actuators A: Physical. pp. 292-297 (2008).

15. Matthew W. Hooker, "Properties of PZT-Based Piezoelectric Ceramics Between-150 and 250 C", Hampton, Virginia, National Aeronautics and Space Administration (1998).

16. M. S. Meetei, A. D. Singh, S. Majumder, "a mathematical modelling and $3 \mathrm{~d}$ analysis of pzt-5h piezoelectric base bridge pressure sensor", International Journal of Mechanical Engineering and Technology. 407-415 (2019). 